\title{
Efficacy, safety, and tolerance of the non-ergoline dopamine agonist pramipexole in the treatment of advanced Parkinson's disease: a double blind, placebo controlled, randomised, multicentre study
}

\author{
M M Pinter, O Pogarell, W H Oertel
}

\begin{abstract}
Objectives-Pramipexole, a non-ergot dopamine D2/D3 receptor agonist, was investigated as an add on drug in advanced parkinsonian patients with motor fluctuations to assess efficacy, safety, and tolerance.

Methods-Seventy eight patients of either sex with advanced Parkinson's disease and treatment complications such as motor fluctuations were enrolled into a double blind, placebo controlled, randomised, multicentre study (phase II) and assigned to add on treatment with pramipexole $(n=34)$ versus placebo $(n=44)$ to a previously stabilised antiparkinsonian medication ( 7 week dose titration interval, 4 week maintenance period). The primary end point of efficacy was the change from baseline in the total score of the unified Parkinson's disease rating scale (UPDRS) in the on "period" ( 2 hours after intake of study medication). Safety and tolerability were assessed on the basis of adverse events, vital signs, laboratory measurements, and ECG recordings.
\end{abstract}

Results-There was a significant improvement of the pramipexole group in UPDRS total scores, subscores part II, III (activities of daily living and motor examination), and IV (complications of therapy). Mean UPDRS total score decreased by $37.3 \%$ under pramipexole compared with $12.2 \%$ under placebo $(p<0.001)$. Patients under pramipexole reported an overall reduction in "off" periods of $12 \%$ resulting in 1.7 more hours "on" time a day-compared with an increase in "off" periods of $2 \%$ under placebo. There were no unexpected safety results. The adverse event profile disclosed a high tolerability. The most important adverse events under pramipexole were fatigue, dyskinesia, and vivid dreams.

Conclusion-Pramipexole administration is an efficacious and well tolerated add on therapy in patients with advanced Parkinson's disease with an improvement in activities of daily living, motor function, and treatment associated complications. (F Neurol Neurosurg Psychiatry 1999;66:436-441)

Keywords: Parkinson's disease, dopamine agonist, pramipexole
Pramipexole, a synthetic aminobenzothiazole derivative, is a non-ergot dopamine agonist with novel properties. It has the highest affinity of the dopamine D2 receptor subfamily and within this group it shows preferential affinity for the D3 receptor subgroup. There is no binding to the dopamine D1 receptor family and apart from dopamine receptors, it binds only to $\alpha 2$-adrenoceptors, but to an extent not clinically relevant. ${ }^{12}$ According to findings in animal models, the binding site of pramipexole on dopaminergic neurons is preferentially located presynaptically, where it acts as an agonist and inhibits dopamine synthesis and release. However, when the presynaptic dopaminergic neuron is impaired, as is the case in Parkinson's disease, pramipexole acts as a potent postsynaptic dopamine D2 receptor agonist. $^{34}$

The pharmacokinetic indices of pramipexole are linear and predictable, plasma concentrations increase proportionally with dosage. Pramipexole is well absorbed after oral administration with a bioavailability of more than $90 \%$ and a half life ranging from 8 to 12 hours. Peak plasma concentrations occur within 1 to 3 hours. Pramipexole undergoes minimal metabolism and is excreted virtually unchanged in the urine. ${ }^{5}$

Pramipexole has been investigated in preliminary studies in early and advanced Parkinson's disease. ${ }^{6-10}$ Main findings in patients with advanced Parkinson's disease with motor fluctuations have been that pramipexole improved motor functions, reduced the time of "off" periods, and decreased the disability and Parkinson's disease severity during "on" and “off" periods..$^{10}$

The purpose of this prospective, double blind, placebo controlled, randomised, multicentre clinical trial (phase II) was to compare the efficacy and tolerability of pramipexole as an add on drug with that of placebo in advanced Parkinson's disease, and to assess the effect of pramipexole on complications associated with levodopa treatment such as motor fluctuations or abnormal involuntary movements.

\section{Patients and methods}

PATIENTS

In this trial, patients with idiopathic Parkinson's disease classified according to the UK Parkinson's Disease Society Brain Bank ${ }^{11}$; who experienced motor fluctuations or abnormal involuntary movements on a stable levodopa 
regimen, were enrolled at nine study centres. Included were patients with severity of Parkinson's disease corresponding to Hoehn and Yahr classification stages II and IV. ${ }^{12}$ The use of concomitant antiparkinsonian drugs such as MAO-B inhibitors and amantadines was allowed, but - as with levodopa (plus decarboxylase inhibitor)-dosages had to remain unchanged during the trial. Excluded were female patients of child bearing potential (contraceptives were not allowed), patients with Parkinson's disease caused by other neurodegenerative diseases, and patients with severe dementia, epilepsy, previous neurosurgery, or severe physical diseases. The concomitant treatment with dopamine agonists, MAO-A inhibitors, neuroleptics, $\alpha$-methyldopa, clonidine, reserpine, and calcium antagonists was not allowed. The study was approved by local ethics committees and written informed consent was obtained from all patients.

\section{METHODS}

After a screening period of up to 2 weeks, patients were randomly assigned under double blind conditions to either pramipexole (34 patients) or placebo ( 44 patients). There was a stratification into four groups according to a high $(>600 \mathrm{mg}$ ) or low ( $\leqslant 600 \mathrm{mg}$ ) daily levodopa dose, with or without other antiparkinsonian medication. Daily doses of trial medication were individually adjusted during a 7 week dose titration interval, with doses being increased weekly from $0.2 \mathrm{mg}$ up to $5.0 \mathrm{mg} /$ day $(2 \times 0.1 \mathrm{mg}, 4 \times 0.1,0.25,0.5,0.75,1.0$, and finally $1.25 \mathrm{mg}$ ) followed by a 4 week maintenance period. At the end of the maintenance period, a reduction in dosage followed to gradually withdraw the study medication over the course of 1 week.

Unless otherwise specified, the following assessments were performed at each visit: the unified Parkinson's disease rating scale (UPDRS) including part I (mentation, behaviour, and mood), part II (activities in daily living), part III (motor examination) and part IV (complications of therapy). ${ }^{13}$ The motor examination was assessed in the "on" period, 2

Table 1 Demographics and baseline characteristics

\begin{tabular}{|c|c|c|c|}
\hline & Pramipexole & Placebo & Total \\
\hline Number of patients & $34^{\star}$ & 44 & $78^{\star}$ \\
\hline \multicolumn{4}{|l|}{ Sex: } \\
\hline Men & $20(58.8 \%)$ & $31(70.5 \%)$ & $51(65.4 \%)$ \\
\hline Women & $14(41.2 \%)$ & $13(29.5 \%)$ & $27(34.6 \%)$ \\
\hline Age $(y)$ & $59.3(8.3)$ & $60.7(8.7)$ & $60.1(8.5)$ \\
\hline Duration (y) of Parkinson's disease & $7.8(4.3)$ & $8.5(5.2)$ & $8.2(4.8)$ \\
\hline \multicolumn{4}{|l|}{ Hoehn and Yahr stage: } \\
\hline II & $7(20.6 \%)$ & $13(29.5 \%)$ & $20(25.6 \%)$ \\
\hline III & $22(64.7 \%)$ & $20(45.5 \%)$ & $42(35.9 \%)$ \\
\hline IV & $5(14.7 \%)$ & $11(25.0 \%)$ & $16(20.5 \%)$ \\
\hline UPDRS total score & $53.6(14.0)$ & $50.2(20.0)$ & $51.7(17.6)$ \\
\hline UPDRS part I & $1.5(1.8)$ & $1.1(1.5)$ & $1.2(1.6)$ \\
\hline UPDRS part II & $13.0(4.9)$ & $12.7(7.3)$ & $12.8(6.3)$ \\
\hline UPDRS part III & $33.5(9.1)$ & $30.5(12.2)$ & $31.8(11.0)$ \\
\hline UPDRS part IV & $5.7(4.0)$ & $5.9(3.5)$ & $5.8(3.7)$ \\
\hline \multicolumn{4}{|l|}{ Antiparkinson medication: } \\
\hline \multicolumn{4}{|l|}{ Only levodopa } \\
\hline$\leqslant 600 \mathrm{mg}$ & $5(14.7 \%)$ & $7(15.9 \%)$ & $12(15.4 \%)$ \\
\hline$>600 \mathrm{mg}$ & $4(11.8 \%)$ & $8(18.2 \%)$ & $12(15.4 \%)$ \\
\hline \multicolumn{4}{|l|}{ Levodopa and other } \\
\hline$\leqslant 600 \mathrm{mg}$ & $15(44.2 \%)$ & $16(36.4 \%)$ & $31(39.7 \%)$ \\
\hline$>600 \mathrm{mg}$ & $10(29.4 \%)$ & $13(29.6 \%)$ & $23(29.5 \%)$ \\
\hline
\end{tabular}

${ }^{\star}$ One patient was randomised to both treatment groups. Values are mean (SD) or mean (\%). hours after intake of study medication. Also assessed were the Hoehn and Yahr scale, the Schwab and England scale (best "on" period, worst "off" period within past week before visit) and the Parkinson dyskinesia scale in "on" period using a five point scale $(0=$ normal, 1 =intermittent, 2 =generalised, 3 =moderate, $4=$ incapacitating) for various body regions (head, upper and lower limbs, and trunk). Furthermore, a global clinical assessment scale with respect to efficacy, tolerance, and compliance (ratings=good, fair, unsatisfactory, not assessable) as judged by the investigators at the end of the maintenance period was also employed, as were patient diaries to record duration and severity of disability during waking hours "off" periods. The diaries were dispensed at screening and before the end of maintenance, and were evaluated by the investigator at baseline and at the end of the maintenance period.

The primary end point was the change in the UPDRS total score at the end of the maintenance interval compared with baseline. Secondary end points were changes in UPDRS subscores (parts I-IV), the Schwab and England scale, the Parkinson dyskinesia scale, the patients' diary, and the global clinical assessment at the end of maintenance interval compared with baseline.

Safety and tolerance were assessed on the basis of neurological examinations, blood pressure and pulse rate measurements, ECG, routine laboratory investigations (blood cell count, erythrocyte sedimentation rate, enzymes, glucose, electrolytes, and urinary findings) - evaluated up to eight times throughout the study period-and adverse events (those events reported for the first time during the treatment phase or with higher intensity compared with baseline).

STATISTICAL ANALYSIS

To evaluate differences between the two treatment groups, the Wilcoxon-MannWhitney test was applied to the UPDRS total score and subscores of parts II, III, and IV. The subscore of UPDRS part II was defined as the sum of the averages of the individual "on" and "off" scores for each item. However, the subscores of UPDRS part I were classified in categories (improved, unchanged, and deteriorated) and were computed using the $\chi^{2}$ test. In all tests, a probability level of $\mathrm{p}<0.05$ was considered significant.

An evaluable patient analysis (per protocol), which comprised all patients with complete data for analysis, was performed, as this was a phase II trial. The obtained results were confirmed by an intent to treat (ITT) analysis using the last observation carried forward (LOCF) method. Considered suitable for ITT analysis were all patients with at least one dose of study medication and completion of at least one postbaseline assessment. The results of the ITT efficacy analysis $(n=77)$ are presented, as these differed only marginally from the per protocol analysis $(n=67)$. 
Table 2 Reduction of UPDRS scores and significances (Wilcoxon-Mann-Whitney) for the ITT population (LOCF)

\begin{tabular}{|c|c|c|c|c|c|c|c|}
\hline & \multicolumn{3}{|c|}{ Pramipexole $(n=33)$} & \multicolumn{3}{|c|}{ Placebo $(n=44)$} & \multirow[b]{2}{*}{$p$ Value } \\
\hline & $\begin{array}{l}\text { Mean change } \\
\text { (SD) }\end{array}$ & $\begin{array}{l}\text { Mean \% } \\
\text { change }\end{array}$ & $\begin{array}{l}\text { Median } \\
\text { change }\end{array}$ & $\begin{array}{l}\text { Mean change } \\
(S D)\end{array}$ & $\begin{array}{l}\text { Mean \% } \\
\text { change }\end{array}$ & $\begin{array}{l}\text { Median } \\
\text { change }\end{array}$ & \\
\hline UPDRS total score & $20.1(16.0)$ & 37.3 & 20.0 & $5.9(12.8)$ & 13.1 & 5.25 & 0.0002 \\
\hline UPDRS part I & $0.7(1.6)$ & 27.0 & 0.0 & $-0.1(1.0)$ & -1.0 & 0.0 & $0.128^{\star}$ \\
\hline UPDRS part II & $4.4(4.7)$ & 32.2 & 4.0 & $1.1(3.4)$ & 7.5 & 1.0 & 0.0034 \\
\hline UPDRS part III & $13.2(11.0)$ & 39.4 & 14.0 & $4.5(9.5)$ & 15.3 & 5.0 & 0.0008 \\
\hline UPDRS part IV & $1.8(2.6)$ & 23.2 & 1.0 & $0.4(2.1)$ & 1.2 & 0.0 & 0.0092 \\
\hline
\end{tabular}

` $\chi^{2}$ test for categories "improved; unchanged; deteriorated".

\section{Results}

In total, 78 patients ( 51 men, 27 women; aged 34 to 75 years) were enrolled in the trial. Thirty four patients received pramipexole, 44 placebo. The baseline demographic information of the total population $(n=78)$ is listed in table 1 . The pramipexole and placebo group were comparable for baseline characteristics and no significant discrepancies between study centres were noted. There were no significant differences between the two groups in age, duration of the disease, and baseline UPDRS total scores and subscores. The relative number of patients in Hoehn and Yahr stage III was higher in the pramipexole group, whereas relatively more patients on placebo were in Hoehn and Yahr stages II and IV. Stratification according to levodopa treatment and other antiparkinsonian medication resulted in a comparable representation of each stratum in both treatment groups. The mean levodopa dose at baseline was 537.5 (SD 314.4) $\mathrm{mg} /$ day in the pramipexole group and $592.6(\mathrm{SD} 264.0) \mathrm{mg} /$ day in the placebo group.

One patient was dropped from the ITT efficacy analysis population $(n=77)$, as he had been unintentionally enrolled and randomised twice, firstly in the placebo group, then in the pramipexole group. The consequent adaptation of baseline values in the pramipexole treated group showed only marginal changes. Ten patients (four pramipexole, six placebo) discontinued the study prematurely; two patients for administrative reasons (withdrawal of consent; protocol violation), and eight patients due to adverse events (see adverse event section). Overall, 67 (87\%) patients (29 pramipexole, 38 placebo) completed the study according to the protocol and were considered in the per protocol analysis.

In the maintenance period, 43 patients (64\%) received the maximum dose of $5 \mathrm{mg}$ /day pramipexole or corresponding placebo; the maximum dose level was reached by 16 patients $(55 \%)$ of the pramipexole group and $27(71 \%)$ of the placebo group. The mean daily maintenance dosage was 3.59 (SD 1.79) mg (minimum of $0.4 \mathrm{mg} /$ day) in the pramipexole group and 4.08 (SD 1.52) $\mathrm{mg}$ (minimum of $0.85 \mathrm{mg} /$ day) in the placebo group. The mean levodopa dose at end of the maintenance period was 511.0 (SD 308.8) $\mathrm{mg} /$ day for the pramipexole group, and 583.5 (SD 273.3) $\mathrm{mg} /$ day for the placebo group.

\section{EFFICACY EVALUATION}

In the per protocol as well as in the ITT analysis, there was a significant improvement in both end point measurements - that is, the UPDRS total score and the subscores II to IV with respect to pramipexole in comparison with placebo. The reduction of UPDRS total score by $20.1(37.3 \%)$ under pramipexole versus 5.9 $(13.1 \%)$ under placebo was highly significant $(p<0.001)$. The mean and median changes of the UPDRS total scores, as well as the subscores and the calculated significances for the ITT population, are presented in table 2 . For the UPDRS total scores a significant difference between treatment and placebo was achieved as early as week 1 and sustained to the end of the maintenance period (fig 1). The median change in UPDRS part II subscore (fig 2) and part III subscore (fig 3) from baseline to end of maintenance, shows a significant difference between treatment and placebo.

An improvement in the Hoehn and Yahr staging was found in six patients $(18 \%)$ of the pramipexole group compared with 12 patients $(27 \%)$ in the placebo group. A deterioration was registered in two patients $(6 \%)$ on pramipexole and in four patients $(9 \%)$ in the placebo group. In the remaining patients (25 patients on pramipexole and 28 patients on

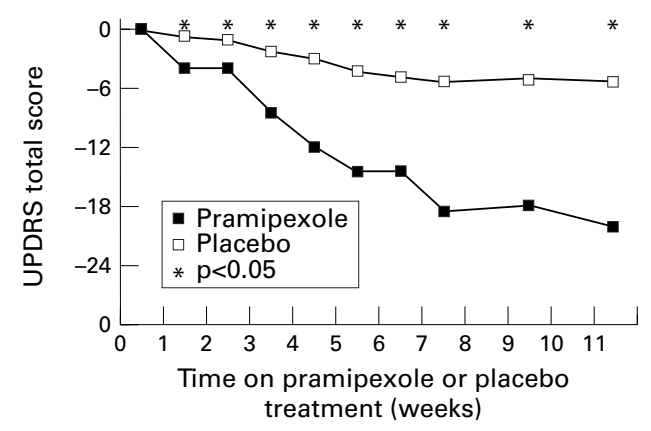

Figure 1 Median change in the UPDRS total score at each visit from baseline to the end of the maintenance period. In comparison with placebo, there is a significant decrease in the UPDRS total scores at each visit $(p<0.05)$ beginning with week 1 .

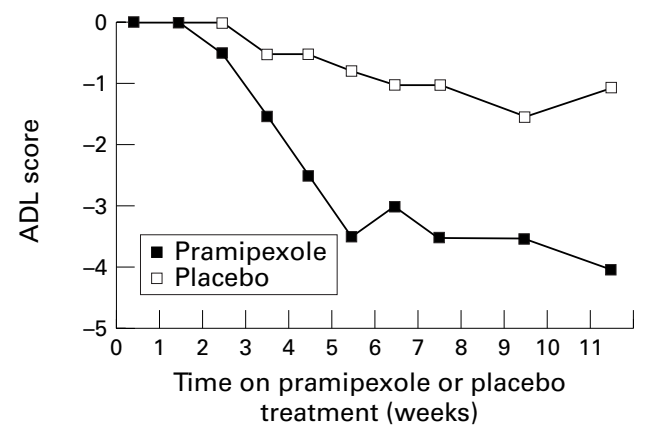

Figure 2 Median change in the subscores of the UPDRS part II (activities of daily living) at each visit from baseline to the end of maintenance period. 


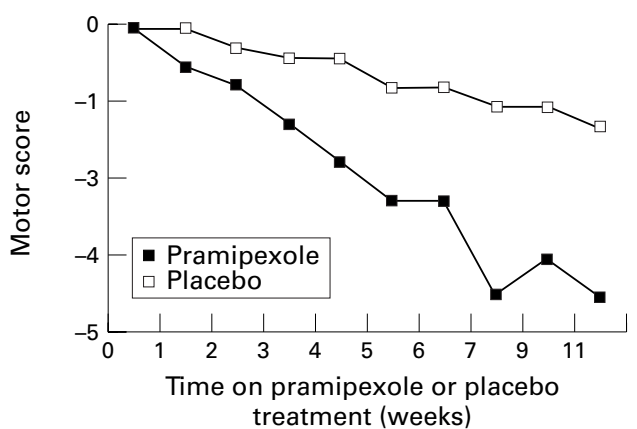

Figure 3 Median change in the subscores of the UPDRS part III (motor scores) at each visit from baseline to the end of maintenance period.

placebo) the baseline and the end of maintenance assessments were identical.

For the Schwab and England scale the following was seen in the pramipexole group: in the "on" period 17 patients (52\%) improved, $16(48 \%)$ remained unchanged, and none worsened, and in the "off" period 18 patients $(54 \%)$ improved, $10(30 \%)$ remained unchanged, four (12\%) deteriorated, and one patient had missing data in the "off" period. In the placebo group, in the "on" period eight (18\%) patients improved, $32(73 \%)$ remained unchanged, and four $(9 \%)$ worsened, whereas in the "off" period $12(27 \%)$ patients improved, $27(62 \%)$ remained unchanged, and five $(11 \%)$ deteriorated. Based on the results obtained with the Schwab and England scale, it is evident that pramipexole treatment was superior compared with placebo; improvement in the "on" period in $52 \%$ of patients versus $18 \%$; in the "off" period in $54 \%$ of patients versus $27 \%$.

Furthermore, pramipexole led to a significant decrease in UPDRS part IV subscore, by about 1.8 (SD 2.6) in the pramipexole group compared with 0.4 (SD 2.1) in those on placebo. However, no significant effect on dyskinesias was found due to pramipexole administration. At the end of the maintenance period, the mean dyskinesia score dropped from 2.70 to 2.12 in the pramipexole group and from 3.70 to 2.77 in the placebo group. In addition, the evaluation of the patients' diaries disclosed that patients on pramipexole reported an overall decrease in average daily percentage of "off" periods during waking hours, from $33.0 \%$ to $20.7 \%$, whereas patients on placebo reported an increase in "off" periods from $32.7 \%$ to $34.6 \%$. The pramipexole group had a $12.3 \%$ reduction of "off" time, which resulted in about 1.7 more hours of "on" time each day. Moreover, based on the global clinical assessment, efficacy was judged as good or fair in $76 \%$ of the pramipexole group compared with $32 \%$ of the placebo group.

SAFETY AND TOLERABILITY

Adverse events were reported by 27 of 34 patients $(79 \%)$ in the pramipexole group and by 32 of 44 patients $(73 \%)$ in the placebo group, whereas they were classified as drug related in 17 patients $(50 \%)$ treated with pramipexole and in 20 patients $(45 \%)$ treated with placebo. Moreover, 15 patients (44\%) in the pramipexole group and 16 patients (36\%) in the placebo group required drug therapy due to adverse events. With respect to the two treatment groups $14(41 \%)$ patients treated with pramipexole versus seven $(16 \%)$ patients treated with placebo reported psychiatric adverse events (mainly vivid dreams and visual hallucinations) and 13 patients (38\%) treated with pramipexole versus 11 (25\%) general disorders (such as fatigue and malaise); whereas in the placebo group relatively more patientsnamely, $21(48 \%)$ versus $11(32 \%)$-had adverse events of the central and peripheral nervous system (mainly dizziness and headache) and musculoskeletal disorders (back pain, myalgia, arthralgia). Gastrointestinal system disorders did not differ between treatment groups.

For the most common adverse events - that is, occurring in at least three patients $(8.8 \%$ for the pramipexole group)-a difference in incidence of more than $5 \%$ of pramipexole over placebo was found for fatigue, dyskinesia, insomnia, agitation, postural hypotension, visual haullucinations, vivid dreams, abnormal lacrimation, nocturia, and renal calculus. Dizziness, headache, and aggravated parkinsonism appeared more often in the placebo group (table 3).

Eight patients, three $(9 \%)$ on pramipexole and five $(11 \%)$ on placebo had to be withdrawn from the study due to adverse events. Reasons for discontinuation in the pramipexole group were (1) sedation and tiredness, (2) drowsiness and myoclonia, and (3) hypotension with collapse and confusion. In the placebo group patients were withdrawn due to (1) nausea, dizziness, and absences, (2) restlessness, (3) influenza, drowsiness, and dizziness, (4) arterial hypertension and headache, and (5) right bundle branch block (ECG recording). No deaths or fatal adverse events were reported.

During the trial, $14 \%$ of the patients had clinically relevant abnormal values in the laboratory evaluation, $24 \%$ in the pramipexole group, $11 \%$ in the placebo group. The abnormalities comprised bacteriuria, raised glucose concentrations, decreased plasma

Table 3 Incidence (\%) of most commonly reported treatment emergent adverse events (occurring with an incidence of at least $8 \%$ or a difference $>5 \%$ between treatment groups)

\begin{tabular}{lcc}
\hline & $\begin{array}{c}\text { Pramipexole } \\
(n=34)\end{array}$ & $\begin{array}{c}\text { Placebo } \\
(n=44)\end{array}$ \\
\hline Fatigue & 29.4 & 4.5 \\
Dyskinesia & 14.7 & 4.5 \\
Insomnia & 4.7 & 9.1 \\
Agitation & 11.8 & 6.8 \\
Vivid dreams & 11.8 & 0.0 \\
Postural hypotension & 8.8 & 2.3 \\
Cramps & 8.8 & 4.5 \\
Hypotension & 8.8 & 4.5 \\
Ataxia & 8.8 & 4.5 \\
Nausea & 8.8 & 6.8 \\
Increased sweating & 8.8 & 6.8 \\
Headache & 5.9 & 18.2 \\
Visual hallucinations & 5.9 & 0.0 \\
Abnormal lacrimation & 5.9 & 0.0 \\
Renal calculus & 5.9 & 0.0 \\
Nocturia & 5.9 & 0.0 \\
Somnolence & 5.9 & 9.1 \\
Dizziness & 2.9 & 27.3 \\
Aggravated parkinsonism & 2.9 & 13.4 \\
Back pain & 0.0 & 6.8 \\
\hline
\end{tabular}


potassium concentrations, increased blood creatine kinase, anaemia, and increased erythrocyte sedimentation rate in the pramipexole group, and anaemia, raised white blood cell count, increased erythrocyte sedimentation rate, and glucosuria in the placebo group. None of these values were linked to the study drug treatment by the investigators and all were found to be normalised during follow up. For the vital signs, pramipexole affected neither blood pressure nor pulse rate. ECG deviations were comparable in frequency under pramipexole and placebo.

\section{Discussion}

The results of this multicentre, double blind, placebo controlled trial with pramipexole up to $5 \mathrm{mg} /$ day in patients with advanced Parkinson's disease disclosed an efficacy to improve parkinsonian signs and to decrease disabilities in daily activities as assessed by clinical rating scales. There was a highly significant reduction in the UPDRS total scores and a significant improvement in the activities of daily living (ADL - UPDRS II), as well as in motor function (UPDRS III) under pramipexole in comparison with placebo. This beneficial treatment effect was prolonged, lasting through the whole maintenance period. Efficacy data as assessed by UPDRS were confirmed by the investigators' global clinical assessment at the end of the trial, which also showed superiority of pramipexole compared with placebo. At first sight, this result is surprising, as an adjunct therapy in the best "on" period should not lead to an improvement of clinical scales. In the present study, however, patients were not assessed during their best "on" period, but in a defined "on" period-that is, 2 hours after their last medication. Therefore, the improvement of UPDRS due to pramipexole indicates a more stable and prolonged "on" period compared with placebo.

According to previously published studies on pramipexole in patients with early Parkinson's disease, the beneficial effect on activities of daily living is greater than the improvement in motor function. ${ }^{6}$ However, another study in early Parkinson's disease found evidence that the treatment effects of pramipexole on motor function compared with placebo were more pronounced in patients with worse UPDRS scores at baseline. ${ }^{78}$ Findings of the present study are similar to those seen in other double blind, placebo controlled trials of pramipexole in patients with advanced Parkinson's disease. ${ }^{9}{ }^{10}$ Although all studies in advanced disease showed a significant change in UPDRS activities of daily living $(22 \%-27 \%)$ and UPDRS motor scores (25-35\%) compared with placebo, the present study discloses a larger improvement induced by pramipexole in the aforementioned scores $(32.2 \%$ in activities of daily living, $39.4 \%$ in motor score).

Furthermore pramipexole compared with placebo led to a small but significant reduction in therapy related complications such as motor fluctuations or abnormal involuntary movements (UPDRS IV) and to a reduction in "off" time, which is in agreement with other studies, although the extent of improvement differed between the reports. Nevertheless in the only study comparing pramipexole with another dopamine agonist (bromocriptine), there was indeed a significant reduction in "off" time only under pramipexole and not under bromocriptine. ${ }^{15}$ This additional favourable effect might be due to the pharmacological properties of pramipexole with its rather long elimination half life of around 12 hours. $^{5}$

Dopamimetic drugs currently available often cause severe and intolerable side effects-for example, hypotension or gastrointestinal disturbances - or other, albeit rare, adverse events such as erythromelalgia, retroperitoneal fibrosis, or pleuropulmonary complications, most likely associated with ergot structure and function of dopamine agonists. ${ }^{15-17}$ Pramipexole showed a low side effect profile, was well tolerated and safe, with fatigue, vivid dreams, and dyskinesia as the most prominent adverse events. Visual hallucinations were experienced by two patients $(5.9 \%)$, which compares favourably with the results gained elsewhere..$^{7-9}$ In particular, gastrointestinal tolerability was good in this study, as the incidence of these symptoms did not differ significantly between pramipexole and placebo groups. However, in early Parkinson's disease gastrointestinal symptoms were more pronounced under pramipexole. $^{7}$

Postural hypotension was only slightly more frequent in the pramipexole group; this is in accordance with the early Parkinson's disease studies, in which there were also no major differences between the treatment groups with respect to postural hypotension. ${ }^{67}$

The higher incidence of dyskinesia, which is indeed a major problem of long term therapy of Parkinson's disease, is most likely to be due to the introduction of pramipexole as an add on therapy. As the patients were not allowed to change their previously stabilised antiparkinsonian medication (including levodopa), the higher incidence of dyskinesias compared with placebo might be related to a dopaminergic overstimulation under pramipexole when added to levodopa. Nevertheless, a preliminary study of pramipexole in patients with advanced Parkinson's disease showed that these dyskinesias could be controlled by lowering the level of levodopa medication. ${ }^{9}$

In conclusion, pramipexole is an effective and well tolerated add on therapy in advanced Parkinson's disease with motor fluctuations. Pramipexole improved motor functions, activities of daily living, and reduced "off" time during the waking day which resulted in more hours of "on" time each day.

The following investigators and coinvestigators were involved in the multicentre design of this study: C Albani, Zuerich,Switzerland; B Conrad, Munich, Germany; W Gehlen, Bochum, Germany; J Glass, Neubrandenburg, Germany; CW Hess, Bern, Switzerland; H Koelmel, Berlin, Germany; T Leonhard, Bernau, Germany; W Poewe, Innsbruck, Austria; G Schnaberth, Vienna, Austria.

1 Mierau J, Schneider FJ, Ensinger H, et al. Pramipexole binding and activation of cloned and expressed dopamine D2, D3, and D4 receptors. Eur F Pharmacol 1995;290:29D2,

2 Mierau J, Bechtel WD. SND 919 inhibits dopamine release in vivo and in vitro. Psychopharmacology 1988;96:338. 
3 Robertson GS, Tham CS, Wilson C, et al. In vivo comparisons of the effects of quinpirole and the putative presynaptic dopaminergic agonists B-HT 920 and SND 919 on striTher 1993;264:1344-51.

4 Kreiss DS, Bergstrom BA, Gonzalez AM, et al. Dopamine receptor agonist potencies for inhibition of cell firing correlate with dopamine D 3 receptor binding affinities. Eur $\mathcal{F}$ Pharmacol 1995;277:209-14.

5 Wright CE, Sisson TL, Ichhpurani AK, et al. Steady-state pharmacokinetic properties of pramipexole in healthy volunteers. 7 Clin Pharmacol 1997;37:520-5.

6 Hubble JP, Koller WC, Cutler NR, et al. Pramipexole in patients with early Parkinson's disease. Clin Neuropharmacol 1995;18:338-47.

7 Parkinson Study Group. Safety and efficacy of pramipexole in early Parkinson's disease. A randomized dose-ranging study. ҰAMA 1997;278:125-30.

8 Shannon KM, Bennett JP, Friedman JH, for the Praminexole Study Group. Efficacy of pramipexole, a novel dopamine agonist, as monotherapy in mild to moderate dopamine agonist, as monotherapy in mild to
Parkinson's disease. Neurology 1997;49:724-8.

9 Molho ES, Factor SA, Weiner WJ, et al. The use of pramipexole, a novel dopamine (DA) agonist in advanced Parkinson's disease. $\mathcal{F}$ Neural Transm Park Dis Dement Sect 1995;45:225-30.
10 Liebermann A, Ranhosky A, Korts D. Clinical evaluation of pramipexole in advanced Parkinson's disease: results of double-blind placebo-controlled, parallel-group study.

11 Gibb WRG, Lees AJ. The relevence of the Lewy body to the pathogenesis of idiopathic Parkinson's disease. I Neurol Neurosurg Psychiatry 1988;51:745-52.

12 Hoehn MM, Yahr MD. Parkinsonism: onset, progression and mortality. Neurology 1967;17:427-42.

13 Fahn S, Elton RL, and Members of the UPDRS Development Committee. Unified Parkinson's disease rating scale. In: Fahn S, Marsden CDE, Calne D, et al, eds. NJ: MacMillian Healthcare Information, 1987:153-63.

14 Guttman $M$, and the International PramipexoleBromocriptine Study Group. Double-blind comparison of pramipexole and bromocriptine treatment with placebo in pramipexole and bromocriptine treatment with placebo in

15 Eisler T, Hall RP, Kalaver K, et al. Erythromelalgia-like eruption in parkinsonian patients treated with bromocriperuption in parkinsonian patients
tine. Neurology 1981;31:1368-70.

16 Demonet JF, Rostin M, Dueymes M, et al. Retroperitoneal fibrosis and treatment of Parkinson's disease with high dose of bromocriptine. Clin Neuropharmacol 1986;9:200-1.

17 Melamed S, Braunstein GD. Bromocriptine and pleuropulmonary disease. Arch Intern Med 1989;149:258-9. 